\title{
Some new results for Hasimoto surfaces
}

\author{
Alev Kelleci ${ }^{1}$, Mehmet Bektaș ${ }^{2}$ \\ 1. Department of Mathematics, Firat University, TURKEY, Elazig, E-mail: akelleci@ firat.edu.tr \\ 2. Department of Mathematics, Firat University, TURKEY, Elazig, E-mail: mbektas@ firat.edu.tr
}

Abstract - Let $\sigma=\sigma(s, t)$ be the position vector of a curve $\Gamma$ moving on surface $M$ in $E^{3}$ such that $\sigma=\sigma(s, t)$ is a unit speed curve for all $t$. If the surface $M$ is a Hasimoto surface, then, the position vector $\sigma$ satisfy the following condition

$$
\sigma_{t}=\sigma_{s} \Lambda \sigma_{s s}
$$

also called as smoke ring equation or vortex filament [1]. In that work, we investigate the geometric properties according to Bishop frame of Hasimoto surfaces in Euclidean 3-space. Also, we give some characterization of parameter curves given according to Bishop frame of Hasimoto surfaces.

Keywords - Hasimoto Surface, Euclidean Space, vortex filament, Bishop frame, smoke ring equation.

\section{Introduction}

In [8], Da Rios invoked what is now known as the localized induction approximation to derive a pair of coupled nonlinear equations guiding the time evolution of the torsion and curvature of vortex filament also called smoke ring equations. Additively In 1972, Hasimoto [2] demonstrated that the Da Rios equations may be associated to generate the celebrated nonlinear Schrodinger (NLS) equation of soliton theory and also in this work, he considered a proximity to the selfinduced motion of a thin isolated vortex filament moving without extending in an incompressible fluid. Finally he obtain that if the position vector of vortex filament is $\sigma=\sigma(\mathrm{s}, \mathrm{t})$, then the formula

$$
\sigma_{t}=\sigma_{s} \Lambda \sigma_{s s}
$$

is hold. In [8], the Da Rios equations and their composition, the NLS equation, are derived in a purely geometric manner via a binormal motion of an inextensible curve. In [3], authors discussed on the Hasimoto surface in $\mathrm{E}_{1}^{3}$, where they invastiged its geometric properties and also gave some characterizations of parametric curves of this surface.

In that work, we move the study of Hasimoto surfaces started in [3] into the Minkowski space. First, we investigate the geometric properties according to Bishop frame of Hasimoto surfaces in Euclidean 3-space. Finally, we give some characterization of parameter curves given according to Bishop frame of Hasimoto surfaces.

\section{Preliminiaries}

Let $\mathrm{E}^{3}$ denote the three-dimensional Euclidean space, that is, the real vector space $\mathrm{R}^{3}$ endowed with the Riemann metric

$$
<,>=\left(d \xi_{0}\right)^{2}+\left(d \xi_{1}\right)^{2}+\left(d \xi_{2}\right)^{2}
$$

where $\left(\xi_{0}, \xi_{1}, \xi_{2}\right)$ is rectangular coordinate system of $\mathrm{E}^{3}$. Let $\mathrm{u}$ an arbitrary vector in $\mathrm{E}^{3}$. So, the norm of $\mathrm{u}$ is given by $\|u\|=\sqrt{|\langle u, u\rangle|}$, [7].

Let $\mathbb{I I}$ be a simply-connected domain in $\mathrm{E}^{2}(\mathrm{t} ; \mathrm{s})$ and $\boldsymbol{\sigma}: \mathbb{I I} \rightarrow \boldsymbol{E}^{3}$ an immersion in $\mathrm{E}^{3}$. If $\sigma=\sigma(s, t)$ is a parametrization of surface $M$ in $E^{3}$, then the unit normal vector field $N$ on $M$ is given by 


$$
N=\frac{\sigma_{s} \wedge \sigma_{t}}{\left\|\sigma_{s} \wedge \sigma_{t}\right\|}
$$

where $\sigma_{s}=\partial \sigma / \partial s$, and $\sigma_{t}=\partial \sigma / \partial t_{s} \wedge$ stands for the Euclidean cross product of $\boldsymbol{E}^{3}$ [7].

The metric <,> on each tangent plane of $\mathrm{M}$ is determined by the first fundemantel form

$$
I=<d \sigma, d \sigma>=E d s^{2}+2 F d s d t+G d t^{2}
$$

with differentiable coefficients

Since we have,

$$
E=\left\langle\sigma_{s}, \sigma_{s}\right\rangle, F=\left\langle\sigma_{s}, \sigma_{t}\right\rangle, G=\left\langle\sigma_{t}, \sigma_{t}\right\rangle
$$

$$
\operatorname{det} I=E G-F^{2}
$$

The shape operatör of the immersion is indicated by the second fundamental form

$$
I I=-\langle d N, d \sigma\rangle=e d s^{2}+2 f d s d t+g d t^{2}
$$

with differentiable coefficients

$$
e=\left\langle\sigma_{s s}, N\right\rangle, f=\left\langle\sigma_{s t}, N\right\rangle, g=\left\langle\sigma_{t t}, N\right\rangle
$$

The Bishop frame or parallel transport frame is an alternative approach to defining a moving frame that is well defined even when the curve $\Gamma$ has vanishing second derivative. One can state parallel transport of an orthonormal frame along a curve simply by parallel transporting each component of the frame [5]. The tangent vector and any convenient arbitrary basis for remainder of the frame are used [5,6]. The Bishop frame is expressed as;

$$
\left[\begin{array}{l}
t \\
y \\
z
\end{array}\right]_{s}=\left[\begin{array}{ccc}
0 & k_{1} & k_{2} \\
-k_{1} & 0 & 0 \\
-k_{2} & 0 & 0
\end{array}\right]\left[\begin{array}{l}
t \\
y \\
z
\end{array}\right],
$$

where the set of $\{t, y, z\}$ is called as Bishop trihedra and the functions $\mathrm{k}_{1}$ and $\mathrm{k}_{2}$ are the Bishop curvatures (see for details in [4]).

\section{Main results}

In this section, as we mentioned before, we move the study of Hasimoto surfaces started in [3] into the Minkowski space. So, we would like to give our main aim as following:

Main theorem: Let $\sigma=\sigma(\mathrm{s}, \mathrm{t})$ be the position vector of a curve $\Gamma$ moving on surface $M$ in Euclidean 3-space such that $\sigma=\sigma(\mathrm{s}, \mathrm{t})$ is a unit speed curve for all $\mathrm{t}$. Then the derivatives of followings are satisfied; 


$$
\left[\begin{array}{l}
t \\
y \\
z
\end{array}\right]_{t}=\left[\begin{array}{ccc}
0 & -\left(k_{2}\right)_{s} & \left(k_{1}\right)_{s} \\
\left(k_{2}\right)_{s} & 0 & -\frac{k_{1}^{2}+k_{2}^{2}}{2} \\
-\left(k_{1}\right)_{s} & \frac{k_{1}^{2}+k_{2}^{2}}{2} & 0
\end{array}\right]\left[\begin{array}{l}
t \\
y \\
z
\end{array}\right]
$$

where $\{t, y, z\}$ is the Bishop frame field and the functions $k_{1}$ and $k_{2}$ are the Bishop curvature functions of the curve $\Gamma$ for all $t$.

Proof. We would like to obtain time derivatives of the Bishop frame $\{t, y, z\}$ which is given the form

$$
\begin{gathered}
t_{t}=\alpha y+\beta z, \\
y_{t}=-\alpha t+\gamma z, \\
z_{t}=-\beta t-\gamma y,
\end{gathered}
$$

where $\alpha, \beta$ and $\gamma$ are smooth functions. Fort his aim, we have to find $\{\alpha, \beta, \gamma\}$ non-zero smooth functions $\mathrm{s}$ in terms of the Bishop curvatures $\mathrm{k}_{1}$ and $\mathrm{k}_{2}$. Using compatibility conditions $\mathrm{t}_{\mathrm{ts}}=\mathrm{t}_{\mathrm{st}}$, (3) yield

$$
\begin{gathered}
\alpha_{s}=\left(k_{1}\right)_{t}-k_{2} \gamma, \\
\beta_{s}=\left(k_{2}\right)_{t}+k_{1} \gamma \\
\gamma_{s}=-k_{1} \beta-k_{2} \alpha .
\end{gathered}
$$

Now, we assume that the velocity of the curve is of the form

$$
\sigma_{t}=\lambda t+\mu y+\vartheta z .
$$

On the other hand from imposition of condition $\sigma_{s t}=\sigma_{t s}$, we find the following equalities

$$
\begin{aligned}
\lambda_{s} & =k_{1} \mu+k_{2} \vartheta_{s} \\
\alpha & =k_{1} \lambda+\mu_{s}, \\
\beta & =k_{2} \lambda+\vartheta_{s},
\end{aligned}
$$

One can choose the correspondance fort he surface $\mathrm{M}$ as $\{\lambda, \mu, \vartheta\} \rightarrow\left\{0,-k_{2}, k_{1}\right\}$. Thus, the velocity vector is given by

$$
\sigma_{\mathrm{t}}=\sigma_{s} \times \sigma_{s s}=-k_{2} y+k_{1} z
$$

which is solution of smoke ring equation. Hence, we can rewrite $(4)_{2,3}$ under the correspondance as

Substituting the last equations into (4) 1 gives

$$
\alpha=-\left(k_{2}\right)_{s}, \beta=\left(k_{1}\right)_{s^{*}}
$$

$$
\gamma=-\frac{k_{1}^{2}+k_{2}^{2}}{2} .
$$

Thus, the proof of main theorem is completed.

\section{Some Characterization of Parameter Curves of Hasimoto surfaces}

In this section, we would like to give new characterizations of parameter curves of Hasimoto surfaces in Euclidean 3-spaces.

Theorem. Assume $\sigma=\sigma(\mathrm{s}, \mathrm{t})$ is a Hasimoto surface in $\mathrm{E}^{3}$. Then the followings are satisfied;

i. s-parameter curves of the surface $\sigma=\sigma(\mathrm{s}, \mathrm{t})$ are geodesics,

ii. t-parameter curves of the surface $\sigma=\sigma(s, t)$ are geodesics if and only if

$$
k_{1}\left(k_{1}\right)_{\mathrm{t}}+k_{2}\left(k_{2}\right)_{\mathrm{t}}=0
$$


where, $\mathrm{k}_{1}$ and $\mathrm{k}_{2}$ are Bishop curvature functions of the curve for all $\mathrm{t}$.

Theorem. Assume $\sigma=\sigma(\mathrm{s}, \mathrm{t})$ is a Hasimoto surface in $\mathrm{E}^{3}$. Then the followings are satisfied;

i. s-parameter curves of the surface $\sigma=\sigma(\mathrm{s}, \mathrm{t})$ are asymtotics if and only if $\kappa=0$,

ii. t-parameter curves of the surface $\sigma=\sigma(s, t)$ are asymptotics if and only if

$$
2\left(k_{2}\left(k_{1}\right)_{t}+k_{1}\left(k_{2}\right)_{t}\right)=\left(k_{1}^{2}+k_{2}^{2}\right)^{2}
$$

where, $\mathrm{k}_{1}$ and $\mathrm{k}_{2}$ are Bishop curvature functions of the curve for all $\mathrm{t}$.

Corollary. If s-parameter curves of a Hasimoto surface $\sigma=\sigma(s, t)$ in $E^{3}$ are asymptotics, then the t-parameter curves are also asymptotics.

Corollary. The parameter curves of a Hasimoto surface $\sigma=\sigma(s, t)$ in $\mathrm{E}^{3}$ are lines of curvature if and only if

$$
k_{1}\left(k_{1}\right)_{s}+k_{2}\left(k_{2}\right)_{s}=0 \text {. }
$$

\section{Conclusion}

In this paper we studied the Hasimoto surfaces in Euclidean 3-spaces. Also we obtained the time derivatives of Bishop trihedra $\{t, y, z\}$ of the curve moving on Hasimoto surfaces. After, we obtained some characterizations of parameter curves of Hasimoto surfaces in $\mathrm{E}^{3}$.

\section{References}

[1] Rogers C., Schief W.K., Backlund and Darboux Transformations, Geometry of Modern Applications in Soliton Theory. Cambridge University Press (2002).

[2] H. Hasimoto, A Soliton on a vortex filament. J. Fluid. Mech. 51, 477-485 (1972).

[3] M. Erdogdu M., M. Ozdemir, Geometry of Hasimoto Surfaces in Minkowski 3-Space, Math. Phys. Anal. Geom. (2014) 17: 169-181.

[4] L. R. Bishop, "There is more than one way to frame a curve", Amer. Math. Monthly, Volume 82, Issue 3, 246-251,1975.

[5] B. Bukcu, M. K. Karacan, The Slant Helices According to Bishop Frame, World Academy of Science, Engineering and Technology Vol:3 (2009) 11-20.

[6] S. Y1lmaz, M. Turgut, A new version of Bishop frame and an application to spherical images, Journal of Mathematical Analysis and Applications, 371 (2010) 764-776.

[7] L. P. Eisenhart, A Treatise On The Differential Geometry Of Curves And Surfaces (1909).

[8] L. S. Da Rios, On the motions of an unbounded fluid with a vortex filament of any shape, (in Italian), Rend. Circ. Mat. Palermo 22, 117 (1906). 